\title{
BASIN BOUNDARY COLLISION AS A MODEL OF DISCONTINUOUS CHANGE IN ECOSYSTEMS
}

\author{
JOHN VANDERMEER ${ }^{1,3}$ AND PETER YODZIS ${ }^{2}$ \\ ${ }^{1}$ Department of Biology, University of Michigan, Ann Arbor, Michigan 48109 USA \\ ${ }^{2}$ Department of Zoology, University of Guelph, Guelph, Ontario, Canada NIG 2WI
}

\begin{abstract}
It has long been recognized that gradual secular changes in environmental parameters may lead to discontinuous changes in ecosystems. The dominant model explaining such changes has been the presence of alternative attractors and their basins. We discuss a related model, that of basin boundary collisions, that may also produce discontinuous changes in an environmental variable. The alternative model suggests that the underlying structure of the basins themselves may be gradually changed by the changing environmental factor. Consequently, the basin of the chaotic attractor may disappear, because it intersects the alternative basin; hence the term basin boundary collision. The implications of this model can be dramatic, such as sudden and unpredictable extinctions or dramatic and unexpected changes in ecosystem syndromes. Furthermore, where this model applies, ancillary phenomena such as a lag in time of the ecosystem change or the existence of socalled chaotic transients may be important. Also discussed are the implications of these results in a world in which slowly changing forcing parameters are well known (e.g., gradual increases of $\mathrm{CO}_{2}$ in the atmosphere, and gradual changes in political attitudes about conservation).
\end{abstract}

Key words: basins of attraction; basin boundary collision; chaos; models, discontinuous change in ecosystems; one-dimensional maps; strange attractors.

\section{INTRODUCTION}

Populations and communities often exhibit dramatic shifts in both time and space. Most frequently, such shifts correspond to a correlated shift in some environmental variable. Forests become pastures, because they are burned by humans; aquatic vegetation gives way to terrestrial vegetation at the margin of a lake. Such obvious cause-and-effect relationships make it easy to assume that any discontinuous change in populations or communities will be associated with some causative environmental parameter that had also dramatically changed. With this assumption, it is an easy trap to tacitly assume the obverse: that gradual secular changes in environmental parameters lead to gradual secular changes in populations and communities. Ecologists have long recognized that this is not true. Frequently, gradual secular changes in environmental parameters can also lead to sudden and dramatic changes in population and community variables.

Such discontinuous transformations in population and community characteristics occur in many ecological contexts. It is common in such cases to seek explanations in parallel changes in some environmental parameter. However, such patterns can be equally explained by various forms of bifurcation (May 1977, Guckenheimer and Holmes 1983, Derrick and Metzgar

Manuscript received 24 November 1997; revised 26 May 1998; accepted 20 August 1998; final version received 14 September 1998.

${ }_{3}^{3}$ E-mail: jvander@umich.edu
1991), one of which is the basin boundary collision (Grebogi et al. 1982, 1983, Abraham and Shaw 1988). In this paper, we explore some of the qualitative behavior patterns expected from this specific bifurcation in its many forms. This bifurcation type is capable of producing dramatic qualitative changes in population behavior in the face of small and secular changes in environmental parameters.

For example, consider population density changes shown in Fig. 1. The population is initially chaotic, suddenly jumping into a stable equilibrium at time a. This behavior is followed by oscillations expanding to chaos again, but is then followed by another jump to different oscillations that decay into a single equilibrium point at time $b$. This point ultimately becomes suddenly chaotic again, at time c, but this time over a broader range of population densities. Witnessing similar discontinuous changes in the behavior of a natural population would likely initiate a search for some particular environmental parameter that, itself, suddenly changed at the same times that the population exhibited these dramatic qualitative changes. However, in fact, those population changes were the consequence of a smooth secular change in an environmental parameter, as will be discussed.

A closely related phenomenon is the sometimes-dramatic time lag that occurs between a sudden environmental change and consequent population or community shifts. The opening of the Welland canal permitted the entrance of lampreys into the Great Lakes, but only 


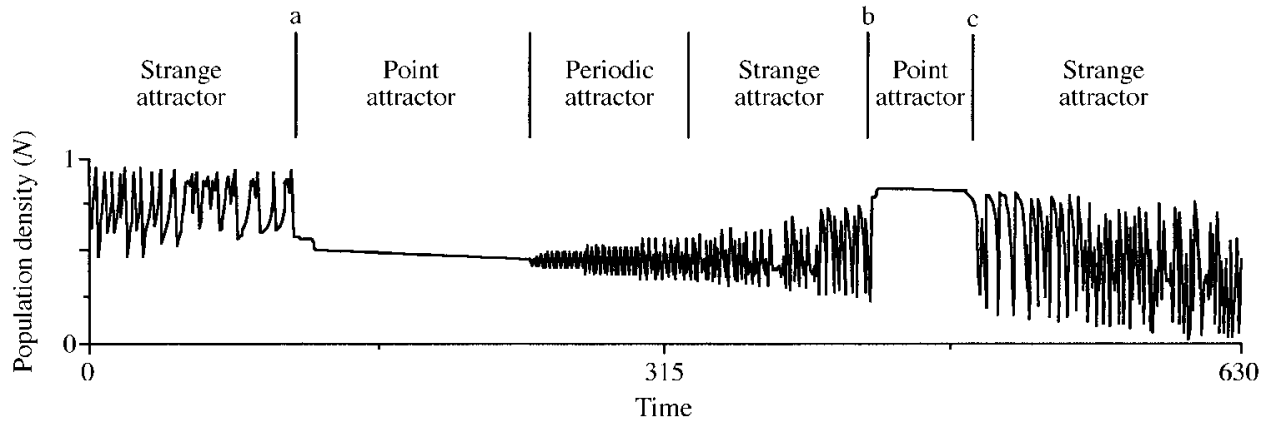

FIG. 1. Exemplary time series of population densities (see Introduction). Note that, throughout, time is measured in time steps that possess no units.

decades later did the dramatic decline of the Great Lakes fishery occur. The inexorable pressure slowly building up from the presence of this novel parasite eventually caused the sudden decline in the commercial fish populations. Similarly, when Europeans migrated westward in North America, they suddenly discovered the delicacy of squabs, thus dramatically altering the habitat and death rate of passenger pigeons. The seemingly infinite abundance of this species suggested that its exploitation could be continued forever, yet its sudden extinction was probably set in stone with the coming of European habits, although the event itself was later in coming.

The prevailing model that is usually employed to explain discontinuous variable change that results from slow secular parameter change is the qualitative idea of alternative attractors with alternative basins (May 1977, Guckenheimer and Holmes 1983, Derrick and Metzgar 1991). The earlier equilibrium state of the Great Lakes fish community may not have been directly altered by the presence of the lamprey, but a new equilibrium point was introduced, one in which the population densities of most commercial fish species was significantly lower than in the original equilibrium point. Thus two equilibrium points theoretically existed, one in which the lamprey population density was zero, and the commercial fish were at exploitable abundances; and one in which the lamprey density was nonzero, and the commercial fish were at relatively lower (and subexploitable) densities. The opening of the Welland Canal pushed the system into the basin of attraction of this second equilibrium point, but it was some time before the population trajectories actually made their way over to the new equilibrium point. In general, alternative equilibria and their basins may normally exist, and environmental variables may push populations such that they suddenly jump from one basin to another, thus resulting in sudden and sometimes dramatic change.

An alternative model suggests that the underlying structure of the basins themselves may be gradually changed by the changing environmental factor. The consequences of this assumption can be dramatic when at least one of the attractors is chaotic. Various dramatic events are possible, such as sudden extinctions (McCann and Yodzis 1994) or dramatic changes in ecosystem syndromes (Vandermeer 1997b). Effectively, there are circumstances in which we expect the basin of the chaotic attractor to effectively disappear, because it intersects the alternative basin; hence the term basin boundary collision. Such an event may occur because of a gradual secular change in an environmental parameter, thus explaining the emergence of sudden dramatic ecosystem change in the face of slow secular parameter change. Furthermore, where this model applies, ancillary phenomena such as a lag in time of the ecosystem change or the existence of so-called chaotic transients may be important (Grebogi et al. 1982, 1983, Abraham and Shaw 1988). This paper seeks to explain such phenomena to a general audience of ecologists, in order that the expected behavior patterns may be more generally appreciated.

In facing a natural world in which gradual environmental changes are certainly the norm, and most recently in which anthropogenic changes are approaching normalcy, an understanding of how such slow secular changes can produce sudden and unexpected changes in population behavior is important. From gradual increases of $\mathrm{CO}_{2}$ in the atmosphere to gradual changes in political attitudes about conservation, such secular changes abound in the contemporary world. While it cannot be denied that these gradual transformations are all around us, can we conclude that ecosystem responses are likely to also be gradual, or might the underlying dynamics of those ecosystems imply sudden qualitative changes in behavior? The basin boundary collision is one mechanism whereby gradual parameter change is translated into sudden and frequently dramatic changes in ecosystem behavior for a variety of ecological models. Exploring this bifurcation form, we use one-dimensional maps for illustrative purposes, especially the logistic map and a particular form of the doubly composed logistic map. However, the results are not particularly sensitive to these particular forms. Basin boundary collisions are common bifurcations in a wide range of continuous models, as well (e.g., Van- 
dermeer 1996). Depending on the context of the model, this bifurcation type may provide a metaphor of qualitative change in ecosystems, generally.

\section{Models \\ Logistic equation with migration}

Simple population growth has frequently been modeled with the logistic equation, either in continuous or discrete form. In most natural populations, the phenomenon of population growth is complicated by migration and/or predator harvesting of some sort. Here we use the logistic map to model such common circumstances. Consider first the simple logistic map depicting the changes in population density, $N$ :

$$
N_{t+1}=r N_{t}\left(1-N_{t}\right)
$$

where $N_{t}$ is the population density at time $t$, and $r$ is the intrinsic rate of increase. The general behavior of this model is now very well understood (May and Oster 1976, Feigenbaum 1979). To make a heuristic point about basins and their boundaries, suppose there is a constant out-migration from the population that is independent of the density of the population. Such a pattern might occur for a variety of reasons. For instance, a constant predator population may extract a constant number of individuals each year, a hunting regulation may permit a constant number of animals to be shot, or a fixed number of fields may be treated with herbicides each year. Assuming density-independent elimination from the population, we modify Eq. 1 to obtain

$N_{t+1}=r N_{t}\left(1-N_{t}\right)-m$ for $r N_{t}\left(1-N_{t}\right)-m>0$

$N_{t+1}=0$

for $r N_{t}\left(1-N_{t}\right)-m \leq 0$

where $m$ is the number of individuals removed from the population during each time unit. There are three singularities, two attractors, and one repeller (two stable equilibrium points and one unstable one), when $m$ $>0$. The upper attractor is given by

$$
\left.N^{*}=(r-1) / 2 r+\left[(r-1)^{2}-4 r m\right)\right]^{1 / 2} / 2 r
$$

while the lower attractor is given by

$$
N^{*}=0 \text {. }
$$

The repeller is given by

$$
\left.N^{*}=(r-1) / 2 r-\left[(r-1)^{2}-4 r m\right)\right]^{1 / 2} / 2 r .
$$

In all cases, the asterisk (*) indicates equilibrium, whether stable or unstable. The repeller is also a separatrix, which is the point that separates the two basins of attraction associated with the two attractors. These concepts are clarified in the diagram in Fig. 2. The basin of attraction (referred to hereafter as simply the basin) is the collection of points such that, when the system is initiated at any of them, it winds up at the associated attractor. The basin is thus aptly compared to a river basin or lake basin, in that all water that falls in the basin, by definition, ends up in the river or lake,

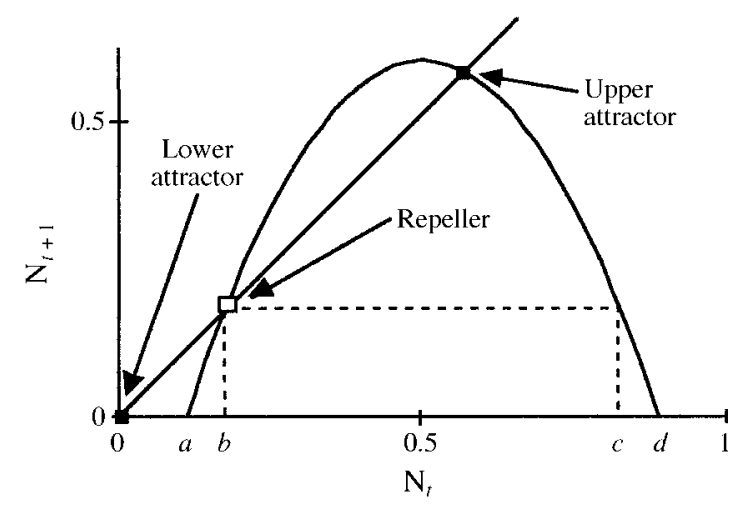

FIG. 2. Graph of Eq. 2 illustrating position of attractors ), repeller $(\square)$, basins, and basin boundaries $(---)$. The regions with $0<N_{t}<b$ and $c<N_{t}<d$ define the basin for the lower attractor, while the region with $b<N_{t}<c$ defines the basin for the upper attractor.

the metaphorical attractor. As is evident in Fig. 2, the single repeller defines the common boundary of the two basins, usually referred to as the separatrix.

This model (Eq. 2) generates the same result reported by McCann and Yodzis (1994) for a different model, namely sudden extinction. The lower attractor of the system represents extinction of the population, and two forms of bifurcation may lead a formerly stable population to that point. The bifurcation diagram with $m$ as the bifurcation parameter, is illustrated in Fig. 3a (for $r=5.0$ ). Note the obvious sudden extinction events: (1) if $m$ slowly increases, when it reaches 0.8 the population suddenly goes extinct, and (2) if $m$ slowly decreases, when it reaches 0.35 the population also suddenly goes extinct. These two extinction events are the result of two distinct bifurcation types, as illustrated in Fig. 3b. The more common bifurcation type occurs when the function, itself, first intersects (i.e., becomes tangent to) the $45^{\circ}$ line, frequently referred to as a saddle-node bifurcation. This point is easily calculated for the present model by setting $N_{t+1}$ equal to $N_{t}$ and solving Eq. $2 \mathrm{a}$ for the case where both roots are equal, thus obtaining the critical value of $m$ (where $m^{*}$ indicates the value of $m$ for which the two roots are equal):

$$
m^{*}=(r-1)^{2} / 4 r
$$

which, for the present example is 0.8 . As we will discuss, structurally a saddle-node bifurcation can be thought of as a special case of the basin boundary collision, in that the repeller, which marks the boundary of the two basins, collides with the upper attractor, which is its own attractor boundary (since it is a single point).

However, a distinct form of basin boundary collision occurs as $m$ decreases to 0.35 , as is evident in the bifurcation diagram (Fig. 3a). Here, the largest projection from a strange attractor (i.e., from the peak of the graph of the function) intersects the boundary of the 

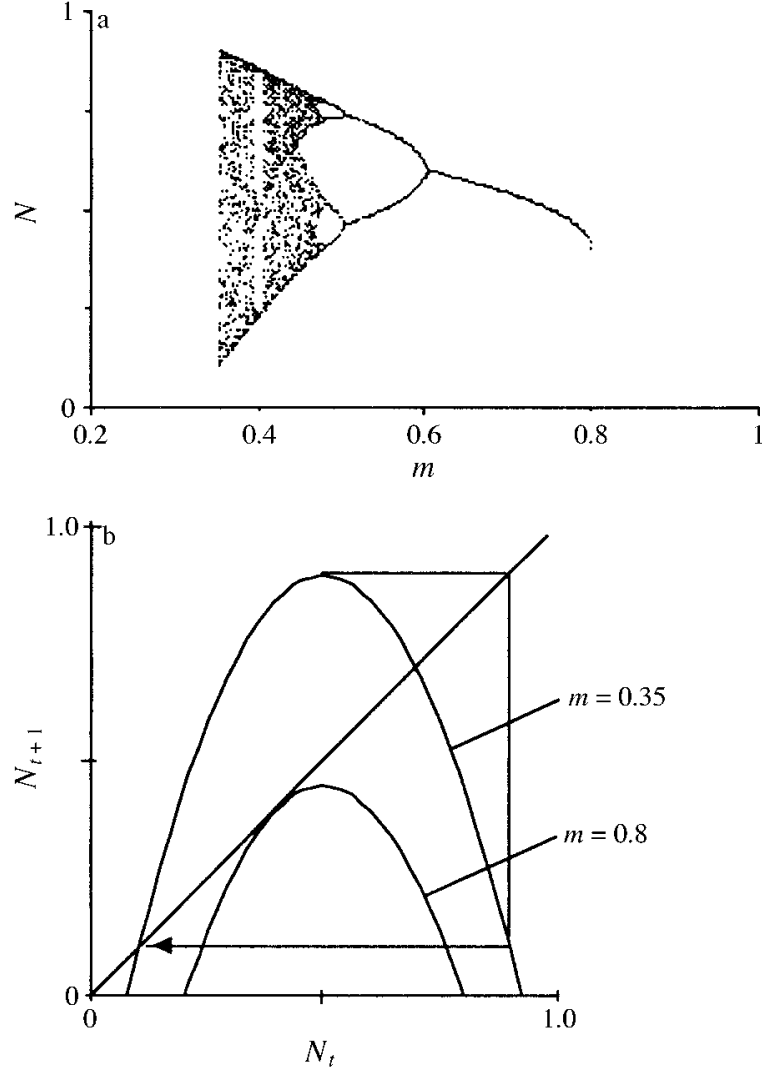

FIG. 3. Analysis of Eq. 2: (a) bifurcation diagram with $r$ $=0.5$; (b) the two basin boundary collisions. The first, occurring at $m=0.35$, is a true basin boundary collision, with the trajectory indicated by the straight-line path that begins at the peak of the function described by Eq. 2 and terminates at the separatrix, as indicated by the arrow. The second, occurring at $m=0.8$, is a saddle-node bifurcation.

basin, thus suddenly incorporating what had been the strange attractor into the basin for the lower attractor. By projecting from the peak of the function, for a given a value of $r$ and solving for $m$ such that projection intersects the lower repeller, it is easy to show that this point occurs when

$$
\begin{aligned}
& (r-1)-\left[(r-1)^{2}-4 m r\right]^{1 / 2} \\
& \quad=2 r^{2}[(r / 4)(1-(r / 4))+r m(m-1)] .
\end{aligned}
$$

Both the saddle-node bifurcation point and the basin boundary collision are illustrated for this example in Fig. 3b. The essential idea of the basin boundary collision is that the projection from the upper boundary of the strange attractor (i.e., the peak of the function) intersects the separatrix, which is the boundary of the lower basin. Thus the trajectories that were formerly members of the strange attractor suddenly become part of the basin, and, in doing so, incorporate all of the space that was previously part of that attractor into the new basin of attraction. The key trajectory is illustrated in Fig. 3b, where it is obvious that the projection from the top of the map (which is the upper boundary of the strange attractor) intersects exactly at the repeller, which marks the position of the basin boundary.

In Fig. 4, we illustrate the dynamic behavior of the model before and after the upper basin boundary collision ( $m=0.35$; see Fig. 3b). In Fig. 4a, the function intersects the $45^{\circ}$ line at two points, both of which are repellers. The zero point is an attractor, and there are two basins. One is associated with the attractor at zero, the other, with the upper repeller; the lower repeller marks a separatrix, separating the two basins. In the upper basin, there is a chaotic attractor, the boundaries of which are shown with dashed lines. In this scenario
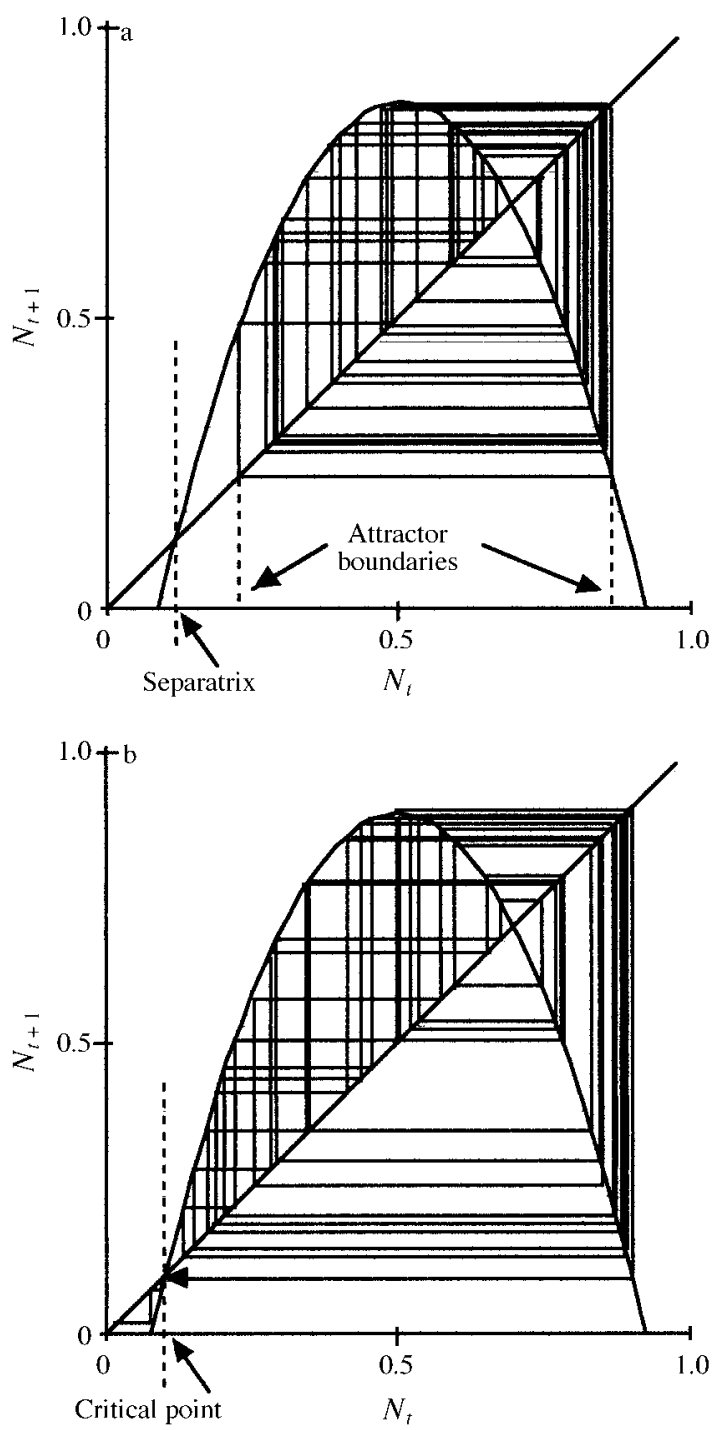

FIG. 4. Illustration of the dynamics of Eq. 2. (a) Two basins of attraction with two attractors (one chaotic and one a point that lies at the origin), with $m=0.0352$. (b) A single basin of attraction with a single attractor (at the origin), but with many trajectories in the part of the basin that had in panel (a) been associated with the chaotic attractor; $m=$ 0.348 . These trajectories are called chaotic transients. 
the system will either remain within the chaotic attractor forever, or will rapidly wind up at zero, depending on where it is initiated. Several trajectories are illustrated within the chaotic attractor.

In Fig. $4 b$, the value of $m$ has been reduced sufficiently to cause the intersection of the separatrix of the two basins with the boundary of the attractor, i.e., the basin boundary collision. This formally obliterates the upper basin since there is no longer an attractor to the right (above) of the lower intersection of the function with the $45^{\circ}$ line (now labeled the "critical point," since it no longer separates two basins and thus cannot be properly called a separatrix). Now, all points of initiation of the system will eventually arrive at zero. However, because the structure of the model is only slightly different from what it was before the basin boundary collision, much of its qualitative behavior looks similar to what it had been. Thus, if the system is initiated to the right of the critical point it will exhibit behavior that looks almost identical to the behavior of the chaotic attractor of Fig. 4a. Nevertheless, eventually the trajectories will breach the critical point, and rapidly converge on zero. The trajectories to the right (above) of the critical point in Fig. 4b have almost identical properties as the trajectories in the chaotic attractors of Fig. 4a. Yet, they are transients (that is, the system inevitably arrives at and stays at zero) and are referred to as "chaotic transients" (Grebogi et al. 1983). Chaotic transients may behave very much like chaotic attractors, but eventually they will suddenly change as the system moves to the region of the basin that had been the alternative basin before the basin boundary collision. For example, in Fig. 4b, the system may behave almost exactly as the system in Fig. 4a for a long period of time, then suddenly, seemingly without warning, it will rapidly go to zero.

It is also worth noting in passing that if $m$ is thought of as the balance between immigration and emigration, reducing the value of $m$ is equivalent to increasing immigration into the population, which, as is evident in the bifurcation diagram (Fig. 3a), tends to promote chaos. This is in contradistinction to the results of Stone (1993) for a more complicated model. Migration may, in this instance, either increase or decrease the likelihood of chaos, depending on the model chosen.

\section{Composed logistic model}

In contradistinction to the previous example, many populations cannot be usefully modeled when all individuals are considered identical. The dramatic difference in survivorship and reproduction due to differences in age for many animal populations is one example. Insects and other invertebrates that progress through different "stages" are another. For such situations, the age- or stage-distributed form of population growth is the appropriate platform (Leslie 1945, Lefkovitch 1965). Here, we note that the fact of a distrib- uted population, when density dependence is operative, is sufficient to generate a basin boundary collision.

Consider a population in two distinct phases, for instance larva $(X)$ and adult $(Y)$. Presuming both larvae and adult live exactly one time period (one time period is necessary for larval development and metamorphosis, and all adults die after one time period), the projection matrix equation would be (Leslie 1945)

$$
\left[\begin{array}{c}
X_{t+1} \\
Y_{t+1}
\end{array}\right]=\left[\begin{array}{ll}
f & 0 \\
0 & p
\end{array}\right]\left[\begin{array}{l}
X_{t} \\
Y_{t}
\end{array}\right] .
$$

A projection matrix with constant coefficients always represents an exponentially growing population. For purposes of the present communication, we expand the model (Eq. 3) to allow for density-dependent effects in both the fecundity factor $(f)$ and the survival probability ( $p$ ) (Higgins et al. 1997). Assume the survivorship factor is

$$
p=r_{1}\left(1-X_{t}\right)
$$

which is to say the larvae undergo a logistic form of survivorship. Maximum survivorship is at the limit as $X_{t}$ approaches zero, and survivorship decreases as a function of the population density of the larvae. Assume the fecundity factor is

$$
f=r_{2}\left(k-Y_{t}\right)
$$

where we presume an upper limit, essentially a carrying capacity, on the population of potential offspring $(k)$, and a maximum rate of offspring production of $r_{2} k$. The resulting matrix equation then becomes

$$
\left[\begin{array}{c}
X_{t+1} \\
Y_{t+1}
\end{array}\right]=\left[\begin{array}{cc}
r_{2}\left(k-Y_{t}\right) & 0 \\
0 & r_{1}\left(1-X_{t}\right)
\end{array}\right]\left[\begin{array}{c}
X_{t} \\
Y_{t}
\end{array}\right]
$$

where, for minimal biological reasons, $r_{1}<1.0$, and $(1 / 4)<k<\left(2\left[r_{2}\right]^{-1 / 2}\right)$. Multiplying the matrix equation, we obtain the following two-dimensional map:

$$
\begin{aligned}
& X_{t+1}=r_{2} Y_{t}\left(k-Y_{t}\right) \\
& Y_{t+1}=r_{1} X_{t}\left(1-X_{t}\right) .
\end{aligned}
$$

This map is conveniently represented as a one-dimensional map through the process of composition, namely,

$$
X_{t+2}=r_{1} r_{2} X_{t}\left(1-X_{t}\right)\left(k-r_{1} X_{t}\left(1-X_{t}\right)\right)
$$

which is a quartic function, the dynamics of which are reasonably well known (Chang et al. 1981, Kot and Shaffer 1984, Vandermeer 1997a).

The first example (Fig. 1) was generated with this model (Eq. 5). The parameter $r_{1}$ was set equal to 1.0 (its maximum value); $k$ and $r_{2}$ were simultaneously varied, with $k$ decreasing and $r_{2}$ increasing, simulating a change along the traditional $r$ and $k$ continuum (holding $r_{1}=1.0, r_{2}$ increasing linearly within 40-50, and $k$ decreasing linearly in the range $0.309-0.25$ ). Thus, one could imagine a rapid but smooth evolutionary change from a species more $k$-like to a species more $r$-like as generating the sorts of sudden changes in de- 

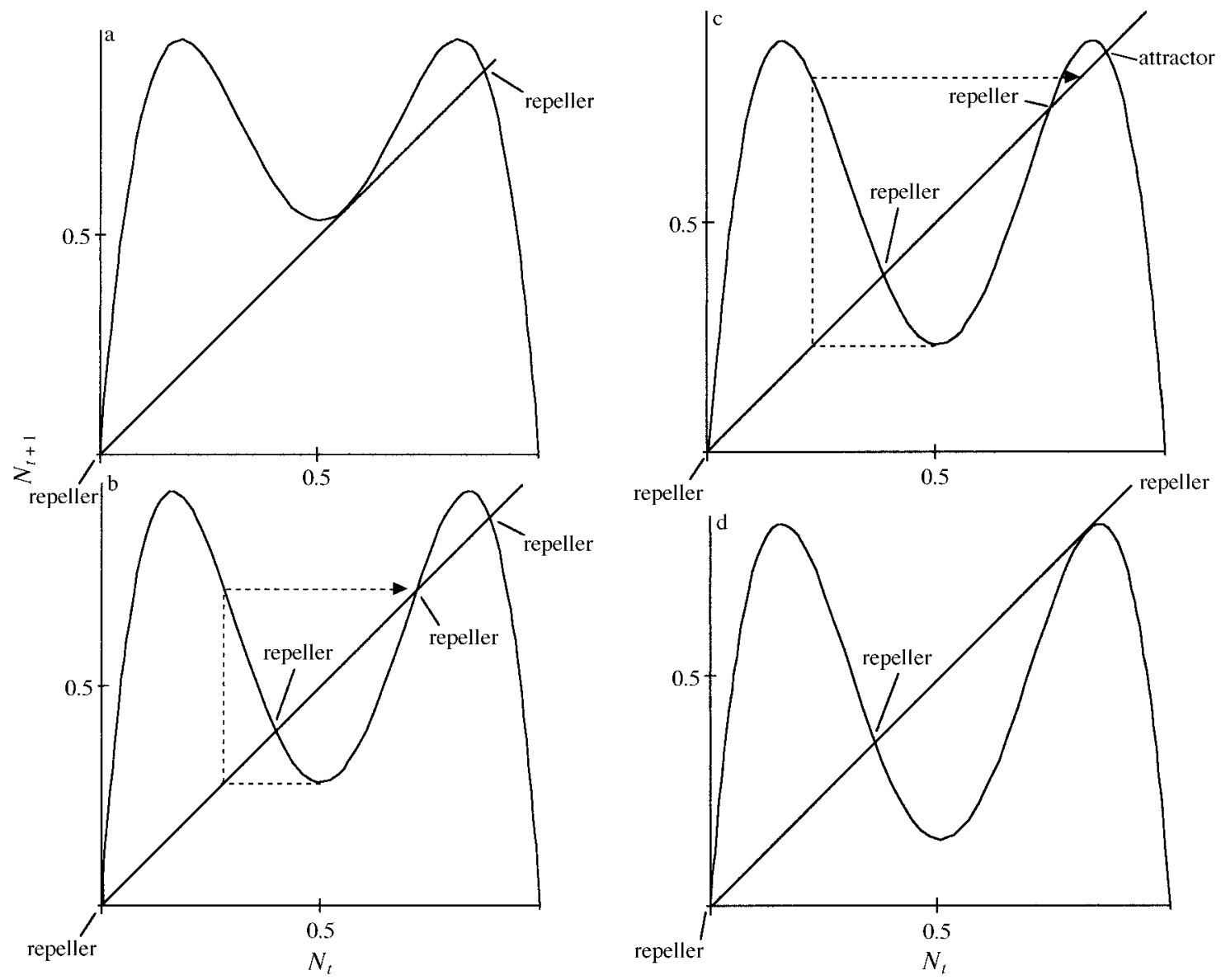

FIG. 5. Illustration of the three bifurcations of Fig. 1. (a) In Fig. 1, point a, $r_{1}=1.0, r_{2}=41.15$, and $k=0.301$. (b) In Fig. 1, point $\mathrm{b}, r_{1}=1.0, r_{2}=46.25$, and $k=0.272$. (c) In Fig. 1, region between points $\mathrm{b}$ and $\mathrm{c}, r_{1}=1.0, r_{2}=47$, and $k$ $=0.267$. (d) In Fig. 1 , point $\mathrm{c}, r_{1}=1.0, r_{2}=48$, and $k=0.262$.

mographic behavior illustrated in Fig. 1. A variety of environmental changes could also be imagined that would generate such smooth transitions of these two parameters.

The three important basin boundary collisions (labeled a, b, and c, in Fig. 1) are illustrated in Fig. 5. In Fig. 5a, the bifurcation point for a saddle-node bifurcation at the lower attractor is presented. The time series in Fig. 1 begins with the function above this point, such that the upper strange attractor is the only attractor in the system. Arriving at the bifurcation point (Fig. 5a, and point a in Fig. 1), the boundary of the strange attractor intersects the basin boundary of the new point attractor created by the saddle-node bifurcation, and the attractor captures all of the trajectories that were formerly associated with the strange attractor. As the parameters $k$ and $r_{2}$ continue changing, the lower point attractor undergoes period doubling bifurcations to another strange attractor (region between a and b in Fig. 1), until the bifurcation point illustrated in Fig. 5b is reached, at which point the basin for the lower attractor intersects that of the upper attractor, which is periodic at this point. Further changes in $k$ and $r_{2}$ cause the upper attractor to change into a point attractor (Fig. 5c) and eventually to undergo another saddle-node bifurcation (Fig. 5d). At this point, what had been the basin of the upper attractor disappears, and the trajectories that had formerly been part of the basin now become part of the lower strange attractor. Again, the saddle-node bifurcation was accompanied by a basin boundary collision in that the basin boundary of the upper point attractor collided with the attractor itself, thus obliterating both basin boundary and point attractor (point $c$ in Fig. 1). Note that there had been trajectories that remained seemingly chaotic during the period between points $b$ and $c$ in Fig. 1. The lower repeller generated complicated trajectories that appeared to be chaotic, but the potential boundaries of what had been a chaotic attractor intersected the basin boundaries of the upper point attractor, and thus those trajectories were formerly just part of the upper basin. With the destruction of that upper basin (at point c in Fig. 1) the chaos-like trajectories become a true strange attractor. This transient behavior is referred to as resulting from chaotic transients, as mentioned earlier (see Models: Logistic equation with migration; (Grebogi et al 1983). 
FIG. 6. Exemplary time series for Eq. 5, with $r_{1}=1.0$ and linear increase in $k$ within the range $0.274-0.268$, and linear increase in $r_{2}$ within the range 49-54.5. (a) Trajectory beginning near the strange attractor at $x \approx 0.5$, and (b) trajectory beginning near the strange attractor at $x \approx 0.75$.
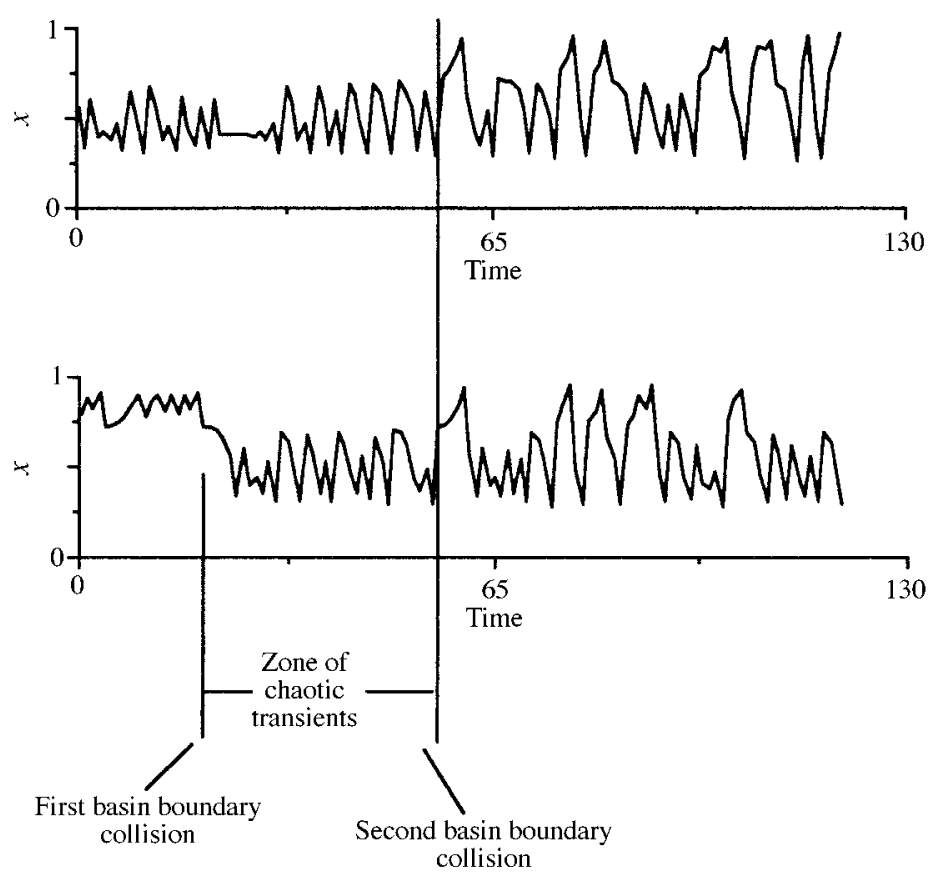

The importance of chaotic transients can be appreciated by the seemingly rare occurrence of strange attractor boundary collisions. If two alternative strange attractors characterize a system, a secular parameter change may move the boundaries of the attractors towards one another, such that at the exact point where one intersects the basin boundary of the other, it also intersects the other's attractor boundary. However, what seems more likely to occur is first the collision of the boundary of one attractor with the basin boundary of the other, and, subsequently, the reverse collision. As an example consider the time series illustrated in Fig. 6. In Fig. 6a, using Eq. 5, a strange attractor located at $x \approx 0.5$ slowly expands with a secular change in $k$ and $r_{2}$ (the exact figures for parameter change are given in the figure legend). Suddenly its boundary collides with the basin of the second, larger attractor, and both the former larger attractor and the smaller attractor become part of the same strange attractor (the time period after the point labeled "second basin boundary collision" in Fig. 6). In Fig. 6b, we begin with the alternative strange attractor, located at $x \approx 0.75$. The same secular change in parameters produces a basin boundary collision (labeled first basin boundary collision in Fig. 6) in which the boundary of this upper attractor collides with the basin boundary of the lower attractor. Furthermore, the former upper attractor loses its identity as an attractor, becoming part of the collection of transients in the expanded basin of attraction of the lower attractor. Further secular changes in the parameter reveals the same behavioral change as observed in Fig. 6a, and at the point of the second basin boundary collision. The exact mechanisms of these two collisions are illustrated in Fig. 7. In Fig. 7a is pictured the first basin boundary collision of Fig. 6. The upper boundary of the upper attractor (the second peak of the map) maps into the basin boundary (the separatrix); thus, that attractor boundary collides with that basin boundary. In Fig. $7 b$ the lower boundary of the lower attractor maps into the middle repeller (which had been the basin boundary before the previous bifurcation), thus uniting the lower attractor with the chaotic transients that had been part of the upper attractor. The terminology here is strained, in that the bifurcation is structurally the same as a basin boundary collision, yet there was no basin boundary to be collided with, since the repeller ceased to mark the boundary of any basin after the first collision had taken place (Fig. 6a). Nevertheless, the basic idea is clear, and inventing a new term would not likely make it clearer.

The general phenomenon is illustrated diagrammatically in Fig. 8. Two separate strange attractors exist in two basins of attraction (Fig 8a). Gradual change in parameters yields the situation in Fig. $8 \mathrm{~b}$, and either the left or right side of the figure. That is, both attractors increase their boundaries, but one does so faster than the other (the left one on the left graph, the right one on the right graph). Eventually, the attractor that increases its boundaries fastest collides with the basin boundary to produce the situation in Fig. 8c. It is easy to conceive of a situation where the left- and righthand sides of the diagram are equal, and both attractors intersect the basin boundary at exactly the same time; this would be called an attractor boundary collision, and would result in a sudden combination of both attractors into one large attractor, more or less encom- 

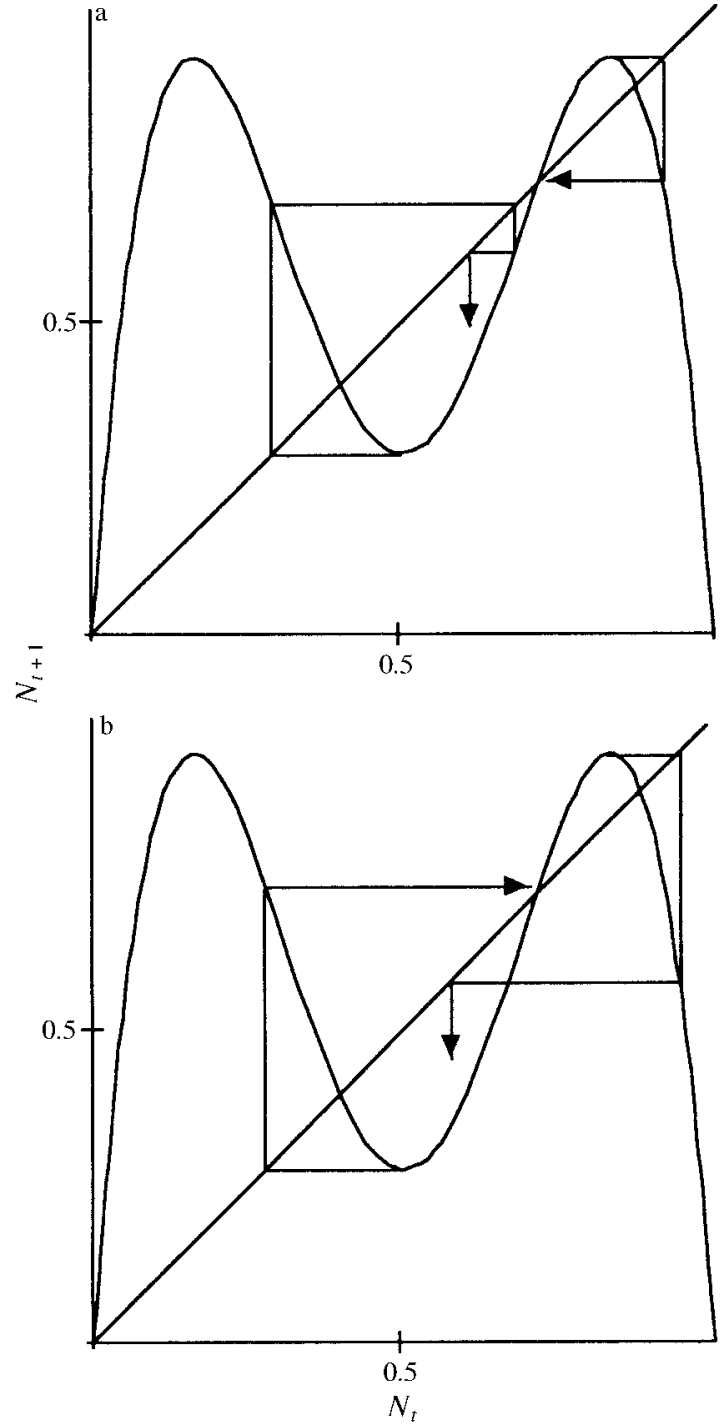

FIG. 7. The critical bifurcations of the time series of Fig. 6. (a) First basin boundary collision (at $r_{2}=49.55$ and $k=$ 0.2734 ); and (b) second basin boundary collision, which involves a shadow attractor (see Models: Composed logistic model) (at $r_{2}=51.2$ and $k=0.2716$ ).

passing the extreme boundaries of both original attractors. Nevertheless, it seems likely that most natural systems would not produce such a collision, but rather one of the attractors will expand more rapidly than the other, resulting in a basin boundary collision, and later a collision with the chaotic transients. It is an important concept, because the chaotic transients form part of the main structure of the large attractor that will be created when the second basin boundary collides with the repeller. Knowing something about the structure of the chaotic transients thus enables a prediction about the large attractor that emerges. Naturally, if the system is arranged so that both basin boundary collisions occur simultaneously (left and right sides of Fig. 8c occur simultaneously) the question of chaotic transients never arises, and we can simply speak of an attractor boundary collision. It seems more likely that basin and attractor boundaries will collide at different times, meaning that usually there will be some time period in which chaotic transients exist.

\section{DISCUSSION}

The phenomenon of discontinuous change in the qualitative behavior of ecosystems is well known, from sudden local extinction of subpopulations (Harrison 1991), to dramatic changes in pollen records (Colinvaux et al. 1996). Previously, discontinuous ecological change has been modeled using the local saddle-node basin boundary collision (May 1977). It is local in the sense that it involves an event at a point in phase space, where a saddle point and a node collide. Here we note that, in nonlinear dynamics, there is a far larger repertoire of global basin boundary collisions that involve events in a finite region of phase space. Thus, discontinuous change in model behavior precipitated by continuous change in model parameters may be far more common in ecological models than one tends to expect.

Noy-Meir's well-known analysis of herbivory (NoyMeir 1975) is an excellent example of alternative basins of attraction and provides a potential example of the operation of basin boundary collisions. Grazing herbivores (and by implication any consumer-resource system) may live in a system with two alternative equilibria and basins: (1) both herbivore and plant densities are high, and the control on the system is from external forces (i.e., the plants are near their carrying capacities, thus control is from "below," due to the competition among individual plants), and (2) both herbivore and plant densities are lower and control is internal to the system (the plant matter is maintained at a very low density by the herbivore pressure, itself, thus control is from "above," due to the consumption by the herbivore). Initiating the system at high grazing densities such that the plants never recuperate fully will result in the second equilibrium, while initiating the system at low grazing densities such that the plants are allowed to approach their own carrying capacity will result in the first equilibrium.

This situation arises when the herbivore isocline is a "humped" isocline, and the predator isocline is sigmoidal in shape, which potentially results from a variety of ecological sources (type III functional response, predator switching behavior, or a tendency of predators to congregate where there are congregations of prey). The two isoclines may thus cross at three points, creating three distinct equilibrium points. The lower point is the control-from-above point, and the upper point is the control-from-below point. The graph in Fig. 9a is such a plot: the lower equilibrium in this case is chaotic, the upper equilibrium is a focal point attractor (damped oscillations), and the middle point is unstable and marks a point on the separatrix between 


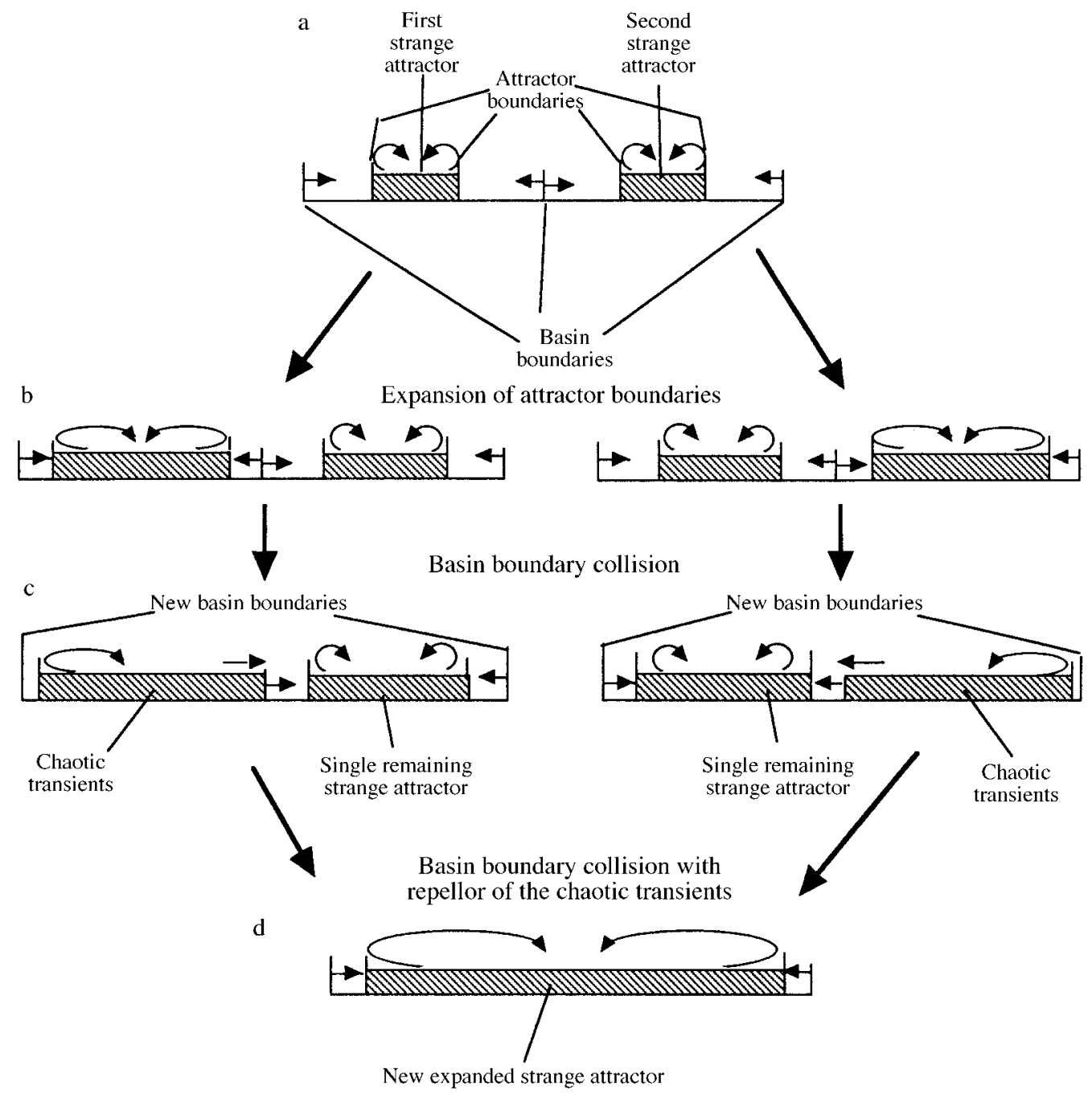

FIG. 8. Diagrammatic representation of the bifurcation sequence of Fig. 6, illustrating two possible pathways to an expanded strange attractor. (a) Two separate strange attractors exist in two basins of attraction. (b) After gradual change in parameters, either the left or right side of the figure emerges. (c) The attractor that increases its boundaries fastest collides with the basin boundary. (d) After further expansion of the boundary of the remaining strange attractor, an expanded strange attractor emerges.

the two basins of attraction (illustrated in this example by a dotted line).

Consider this formulation in terms of the well-known phenomenon of secondary agricultural pests emerging as a consequence of pesticide-induced mortality of natural enemies. The system can be qualitatively modeled as shown in Fig. 9a, where two basins of attraction are associated with two attractors. When concentrated near the lower attractor, the natural enemy holds the pest species under control, which is to say the population density of the pest is always below the economic threshold. In the example of Fig. 9a, and the lower attractor is drawn as a chaotic attractor, but it is a highly constrained chaotic attractor, such that trajectories are contained within a relatively small area around the central unstable equilibrium point. (In a two-dimensional case it is not formally possible to have a chaotic attractor, but we here assume, for heuristic purposes, that there are other variables in the system that provide the necessary multidimensionality for chaos to emerge and that we are simply plotting this particular two-dimensional subsystem). When the system is located at this lower chaotic attractor, the natural enemy is in control of the potential pest, which never exceeds the economic threshold and is probably not even noticed by the farmer. However, after the initiation of pesticide spraying, for some other species of pest that indeed does exceed its economic threshold, the predator isocline will generally decline with an associated distortion in the basins of attraction. The prey isocline is likely to decline also, but usually the predator is more affected by the spray than the herbivore. Thus, here we examine the system 


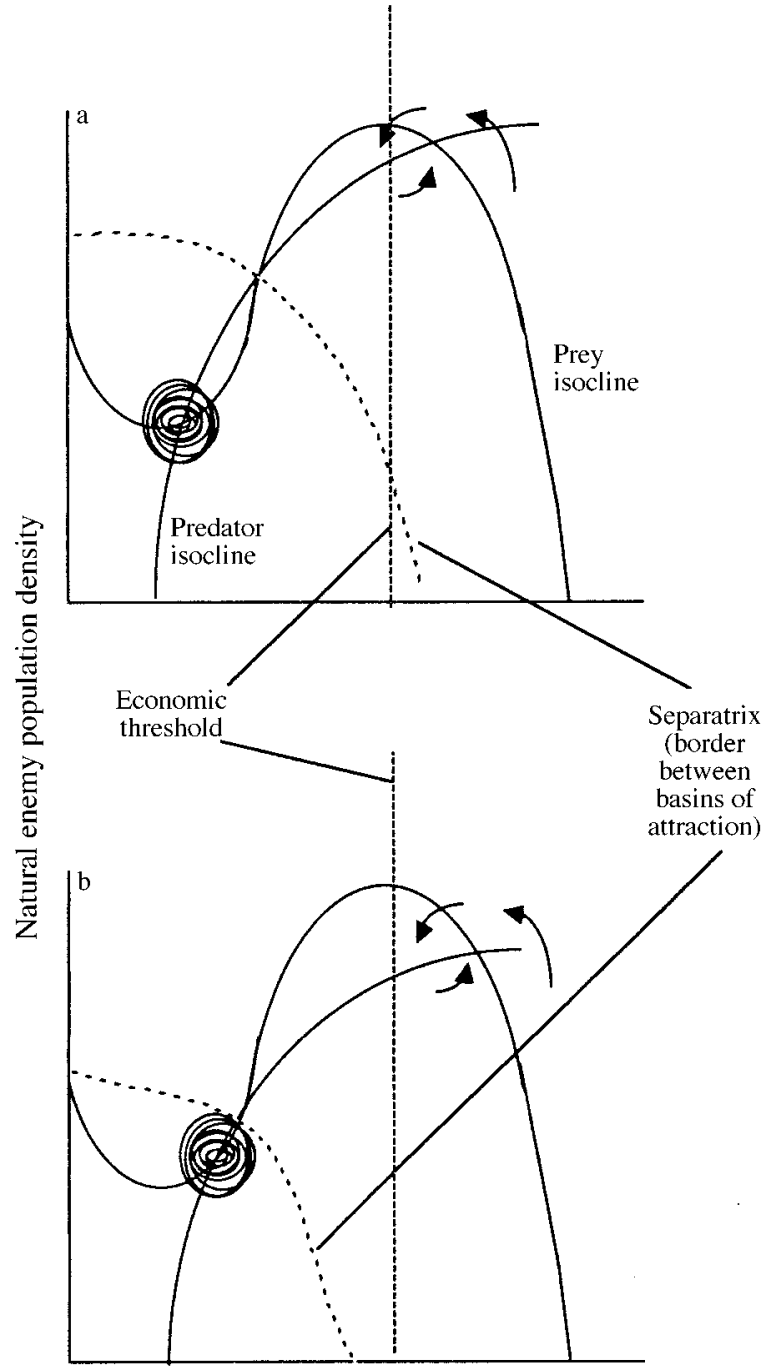

Pest population density

FIG. 9. Illustration of the possible dynamics of a pest species and its natural enemies, using the basic formulation of Noy-Meir (1975), with typical predator and prey isoclines delimiting qualitatively distinct dynamic zones of the phase space. The economic threshold is the minimum size of the pest population that causes significant economic damage (a) Existence of two attractors in two basins of attraction. The lower attractor is chaotic, and the upper attractor is a focal point. The lower attractor, even though chaotic, has boundaries that ensure the pest will never exceed the economic threshold limit. (b) After pesticide application is initiated, the predator isocline is changed, and the resulting separatrix intersects the boundary of the strange attractor. This is the point of basin boundary collision. The chaotic transients, which were formerly simply the trajectories of the chaotic attractor in panel (a), may keep the system below the economic threshold for some long period of time, but eventually the trajectory closest to the boundary of the attractor will be realized. At this point, the other basin of attraction will attract the trajectory, and it will rapidly move to the other attractor (which is a focal point attractor) and maintain itself forever above the economic threshold. The pest has thus become a secondary pest. with only the predator isocline changing. The new situation, after pesticide spraying has begun, is illustrated in Fig. 9b. Here the separatrix has encountered the boundary of the strange attractor, and the lower basin of attraction merges with the upper basin. The pest now will inevitably exceed the threshold level, although it may stay within the bounds of what had before been its chaotic attractor for a long period of time. That is, it may exhibit many chaotic transients, never exceeding the economic threshold, before actually moving up to the higher attractor and becoming a legitimate pest. The amount of time it spends within the confines of the original attractor boundaries (the time it exhibits chaotic transients) could be very large, implying that the initiation of pesticide spraying could have set in stone the fact that this potential pest will someday become a real pest, and it is only a matter of time before it emerges as such.

The many types of discontinuities found in nature could well be fingerprints of basin boundary collisions. For example, vegetation successional sequences can exhibit discontinuous changes at some point during succession. Mortality rates of Eryngium cuneifolium, a fire-dependent Florida scrub, increased dramatically $\sim 6-7$ yr subsequent to fire (Menges and Kimmich 1996). Postfire succession caused a gradual change in microhabitat parameters that may have resulted in a basin boundary collision between the species' attractor and the extinction basin. The parameter $m$ in Eq. 1 may be thought of as a metaphor for succesional change, and, as it increases, the extinction basin boundary moves upwards, eventually colliding with the attractor.

Various zonations, so well known in ecology, could also be consequences of this sort of bifurcation. For example, Vaccinium myrtillus and Vaccinium uliginosum exhibit starkly discontinuous distributions on a combined $\mathrm{pH}$ and water table depth gradient (Bragazz and Gerdol 1996). Gradual changes in $\mathrm{pH}$ and water table depth may represent environmental changes that eventually induce a basin boundary collision, thus explaining this discontinuity.

The use of some groups as bioindicators of ecological stress may be misleading if there are alternative attractors and there exists the potential for basin boundary collisions. Observed changes in the bioindicators used to calibrate this tool may represent slow changes in one attractor and ignore the possible existence of an alternative attractor, leading to an underestimate of the potential for ecosystem change with further environmental perturbation. The potential existence of alternative attractors and the possibility of basin boundary collisions should reinforce current reticence to apply predictive models beyond the empirical range of parameter estimates (Weiner 1996).

The potential for basin boundary collision to cause qualitative change in ecological equilibria is clear. While other mechanisms may equally account for such change, either through the dynamics of population in- 
teractions or the physical background itself being discontinuous, basin boundary collision is an additional phenomenon that should be added to the collection of potential mechanisms. Furthermore, the basin boundary collision also predicts the possibility that dramatic changes in population behavior can happen with no change whatsoever in parameters. That is, once the basin boundary collision has occurred, the population trajectories may continue as chaotic transients for a long period of time before stabilizing at the point in the alternative basin. Thus, to take the previous pest example, after the initiation of pesticide spray, there may be no further change in the ecosystem. Yet, eventually the previously benign herbivore will eventually surge to become a pest, a fact that may seem enigmatic to an observer not aware that there had been a basin boundary collision in the past.

\section{ACKNOWLEDGMENTS}

We would like to thank Joe Scanio, Beverly Rathcke, Kim Cuddington, and Kevin McCann for thoughtful comments on the manuscript.

\section{Literature Cited}

Abraham, R. H., and C. D. Shaw. 1988. Dynamics-the geometry of behavior. Part 4: Bifurcation Behavior. Aerial Press, Santa Cruz, California, USA.

Bragazz, L., and R. Gerdol, 1996. Response surfaces of plant species along water-table depth and $\mathrm{pH}$ gradients in a poor mire on the southern Alps (Italy). Annales Botanici Fennici 33:11-20.

Chang, S., M. Wortis, and J. A. Wright. 1981. Iterative properties of a one-dimensional quartic map: critical lines and tricritical behavior. Physical Review A 24:2669-2684.

Colinvaux, P. A., P. E. De Oliveira, J. E. Moreno, M. C. Miler, and M. B. Bush. 1996. A long pollen record from lowland amazonia: forest and cooling in glacial times. Science $\mathbf{2 7 4}$ $85-88$.

Derrick, W., and L. Metzgar. 1991. Dynamics of LotkaVolterra systems with exploitation. Journal of Theoretical Biology 153:455-468.

Feigenbaum, M. J. 1979. The universal metric properties of nonlinear transformations. Journal of Statistical Physics 19: $25-52$.

Grebogi, E., E. Ott, and J. A. Yorke. 1982. Chaotic attractors in crisis. Physical Review Letters 48:1507-1510.

Grebogi, E., E. Ott, and J. A. Yorke. 1983. Crises, sudden changes in chaotic attractors and transient chaos. Physica D 7:181-200.

Guckenheimer, J., and P. Holmes. 1983. Nonlinear oscillations, dynamical systems, and bifurcations of vector fields. Springer-Verlag, New York, New York, USA.

Harrison, S. 1991. Local extinction in a metapopulation context: an empirical evaluation. Pages 73-88 in M. Gilpin and I. Hanski, editors. Metapopulation dynamics: empirical and theoretical investigations. Academic Press, London, UK.

Higgins, K., A. Hastings, and L. W. Botsford. 1997. Density dependence and age structure:: nonlinear dynamics and population behavior. American Naturalist 149:247-269.

Kot, M., and W. M. Schaffer. 1984. The effects of seasonality on discrete models of population growth. Theoretical Population Biology 26:340-360.

Lefkovitch, L. P. 1965. An extension of the use of matrices in population mathematics. Biometrics 22:1-18.

Leslie, P. H. 1945. On the use of matrices in certain population mathematics. Biometrika 33:183-212.

May, R. M. 1977. Thresholds and breakpoints in ecosystems with a multiplicity of stable states. Nature 269:471-477.

May, R. M., and G. Oster. 1976. Bifurcations and dynamic complexity in simple ecological models. American Naturalist 110:573-599.

McCann, K., and P. Yodzis. 1994. Nonlinear dynamics and population disappearances. American Naturalist 144:873879.

Menges, E. S., and J. Kimmich. 1996. Microhabitat and timesince-fire: effects on demography of Eryngium cuneifolium (Apiaceae), a Florida scrub endemic plant. American Journal of Botany 8:185-191.

Noy-Meir, I. 1975. Stability of grazing systems: an application of predator-prey graphs. Journal of Ecology 63:459481.

Stone, L. 1993. Period-doubling reversals and chaos in simple ecological models. Nature 365:617-620.

Vandermeer, J. H. 1996. Seasonal isochronic forcing of Lotka Volterra equations. Progress of Theoretical Physics 96: $13-28$.

Vandermeer, J. H. 1997a. Period "bubbling" in simple ecological models: pattern and chaos formation in a quartic model. Ecological Modelling 95:311-317.

Vandermeer, J. H. 1997b. Syndromes of production: an emergent property of simple agroecosystem dynamics. Journal of Environmental Management 51:59-72.

Weiner, J. 1996. Problems in predicting the ecological effects of elevated $\mathrm{CO}_{2}$. Pages 431-441 in C. Korner and F. A. Bazzaz, editors. Carbon dioxide, populations, and communities. Academic Press, New York, New York, USA. 